\title{
Melanogenesis Inhibitory, Anti-Inflammatory, and Chemopreventive Effects of Limonoids from the Seeds of Azadirachta indicia A. Juss. (Neem)
}

\author{
Toshihiro Akihisa ${ }^{1 *}$, Taisuke Noto ${ }^{1}$, Akitomo Takahashi ${ }^{1}$, Yukiko Fujita ${ }^{2}$, \\ Norihiro Banno ${ }^{2}$, Harukuni Tokuda ${ }^{3}$, Kazuo Koike ${ }^{4}$, Takashi Suzuki ${ }^{5}$, \\ Ken Yasukawa ${ }^{5}$ and Yumiko Kimura ${ }^{5}$ \\ ${ }^{1}$ College of Science and Technology, Nihon University (1-8 Kanda Surugadai, Chiyoda-ku, Tokyo 101-8308, JAPAN) \\ ${ }^{2}$ Ichimaru Pharcos Company Ltd. (318-1 Asagi, Motosu-shi, Gifu 501-0475, JAPAN) \\ ${ }^{3}$ Department of Complementary and Alternative Medicine, R\&D, Graduate School of Medical Science, Kanazawa University (13-1 Takara- \\ machi, Kanazawa 920-8640, JAPAN) \\ ${ }^{4}$ School of Pharmaceutical Sciences, Toho University (2-2-1 Miyama, Funabashi-shi, Chiba 274-8510, JAPAN) \\ ${ }^{5}$ College of Pharmacy, Nihon University (7-7-1 Narashinodai, Funabashi-shi, Chiba 274-8555, JAPAN)
}

\begin{abstract}
Thirty-one nortriterpenoids, including 28 limonoids (1-28) and 3 degraded limonoids (29-31), and one diterpenoid (32), were isolated from the seed extract of Azadirachta indica (neem). Among these, six were new compounds and their structures were established to be 15-hydroxyazadiradione (3), 7 benzoyl-17-hydroxynimbocinol (5), 23-deoxyazadironolide (12), limocin $E$ (13), 23-epilimocin $E$ (14), and $7 \alpha$-acetoxy-3-oxoisocopala-1,13-dien-15-oic acid (32). Upon evaluation of compounds 1-32 on the melanogenesis in the B16 melanoma cells, five compounds, 20, 26, 27, 29, and 31, exhibited marked inhibitory effect $(74-91 \%$ reduction of melanin content at $25 \mu \mathrm{g} / \mathrm{mL}$ ) with no or almost no toxicity to the cells. Seven compounds, $1,6,9,10,18,20$, and 26, on evaluation for their inhibitory effect against 12- $O$ tetradecanoylphorbol-13-acetate (TPA)-induced inflammation $(1 \mu \mathrm{g} / \mathrm{ear})$ in mice, exhibited, except for compound 26, marked anti-inflammatory activity ( $\mathrm{ID}_{50}$ values $0.09-0.26 \mathrm{mg} / \mathrm{ear}$ ). In addition, all of the 32 compounds exhibited moderate or potent inhibitory effects ( $\mathrm{IC}_{50}$ values of 230-501 mol ratio/32 pmol TPA) against the Epstein-Barr virus early antigen (EBV-EA) activation induced by TPA. Furthermore, on evaluation of azadirachtin $B$ (21) for its anti-tumor-initiating activity on the two-stage carcinogenesis of mouse skin tumor induced by peroxynitrite (ONOO-; PN) as an initiator and TPA as a promoter, this exhibited marked inhibitory activity.
\end{abstract}

Key words: Azadirachta indicia, neem, limonoids, antimelanogenesis activity, anti-inflammatory activity, chemopreventive effect

\begin{abstract}
1 INTRODUCTION
The neem tree (Azadirachta indica A. Juss.; Meliaceae) is indigenous to India, and now this tree is cultivated widely in tropical areas of the world ${ }^{11}$.Various parts of the neem tree have been used for food, medicine, and insecticides since ancient times, and many bioactive constituents including tetranortriterpenoids (limonoids) have been isolated and identified from various parts of neem tree ${ }^{1-4)}$. In the course of our search for potential bioactive compounds from natural sources ${ }^{5}$, we were especially interested to
\end{abstract}

undertake the investigation of $A$. indica. In this paper, we present the isolation and structure elucidation of five new limonoids (3, 5, 12-14) and one new diterpenoid (32) along with 26 known limonoids from a $\mathrm{MeOH}$ extract of defatted seeds of $A$. indica. In addition, we present the evaluation of their inhibitory effects on melanogenesis in the B16 melanoma cells, on $12-O$-tetradecanoylphorbol-13-acetate (TPA)-induced inflammation in mice, and on Epstein-Barr virus early antigen (EBV-EA) activation induced by TPA. Further, we report the inhibitory effect of azadirachtin $B$

${ }^{*}$ Correspondence to: Toshihiro Akihisa, College of Science and Technology, Nihon University, 1-8 Kanda Surugadai, Chiyoda-ku, Tokyo 101-8308, JAPAN E-mail: akihisa@chem.cst.nihon-u.ac.jp

Accepted July 15, 2009 (received for review June 25, 2009)

Journal of Oleo Science ISSN 1345-8957 print / ISSN 1347-3352 online

http://www.jstage.jst.go.jp/browse/jos/ 


\section{T. Akihisa, T. Noto, A. Takahashi et al.}

(21) on in vivo two-stage mouse-skin carcinogenesis.

\section{EXPERIMENTAL}

\subsection{General experimental procedures}

Optical rotations were measured on a JASCO DIP-370 Digital Polarimeter (EtOH; $25{ }^{\circ} \mathrm{C}$ ). UV spectra, using a Shimadzu UV-2200 spectrometer, and IR spectra, using a JASCO FTIR-300E spectrometer, were recorded in EtOH and $\mathrm{KBr}$ disks, respectively. NMR spectra were recorded with a JEOL ECX-500 $\left({ }^{1} \mathrm{H}, 500 \mathrm{MHz} ;{ }^{13} \mathrm{C}, 125 \mathrm{MHz}\right)$ or with a JEOL ECA-600 $\left({ }^{1} \mathrm{H}, 600 \mathrm{MHz} ;{ }^{13} \mathrm{C}, 150 \mathrm{MHz}\right)$ spectrometer in $\mathrm{CDCl}_{3}$ with tetramethylsilane as an internal standard. ESIMS and high-resolution (HR)-ESIMS were recorded with a positive-ion mode on an Agilent $1100 \mathrm{LC} / \mathrm{MSD}$ TOF system. Silica gel (Silica gel 60, 220-400 mesh, Merck) and $\mathrm{C}_{18}$ silica (ODS; Chromatorex-ODS, 100-200 mesh; Fuji Silysia Chemical, Ltd., Aichi) were used for open column chromatography. Reversed-phase preparative HPLC was carried out on ODS columns ( $25 \mathrm{~cm} \times 10 \mathrm{~mm}$ i.d.) with the flow rate of $3.0 \mathrm{~mL} / \mathrm{min}$ of mobile phases, on a Pegasil ODS-II $5 \mu \mathrm{m}$ column (Senshu Scientific Co., Ltd., Tokyo) with $\mathrm{MeCN} / \mathrm{H}_{2} \mathrm{O} / \mathrm{AcOH}$ (50:50:1) (HPLC system I), $\mathrm{MeCN} / \mathrm{H}_{2} \mathrm{O}$ (1:1) (HPLC system II), $\mathrm{MeOH} / \mathrm{H}_{2} \mathrm{O}$ (11:9) (HPLC system III), or with $\mathrm{MeOH} / \mathrm{H}_{2} \mathrm{O}$ (7:3) (HPLC system IV) as the mobile phase, or on a Hypersil ODS-5 (Senshu Scientific Co., Ltd.) with $\mathrm{MeOH} / \mathrm{H}_{2} \mathrm{O}$ as the eluting solvent at a ratio of 3:2 (HPLC system V) or 9:11 (HPLC system VI).

\subsection{Materials}

Oven-dried fruits of Azadirachta indica A. Juss. (Meliaceae), cultivated in Tamil Nadu province of India, were obtained in September, 2005. The plant material was authenticated by one (N. B.) of the authors, and a voucher specimen (No. SH0603-1511) has been deposited in the Research Laboratory, Ichimaru Pharcos Co. Ltd.

\subsection{Chemicals and reagents}

Chemicals and reagents were obtained from the following sources: Fetal bovine serum (FBS) from Trace Scientific Ltd (Melbourne, Australia), Eagle's minimal essential medium (MEM) from Gibco (Grand Island, NY), arbutin (4hydroxyphenyl $\beta$-D-glucopyranoside) from Tokyo Kasei Kogyo Co., Ltd. (Tokyo), TPA from ChemSyn Laboratories (Lenexa, KS), thiazolyl blue tetrazolium bromide (MTT), indomethacin, and (all-trans)-retinoic acid from Sigma Chemical Co. (St. Louis, MO), and the EBV cell culture reagents and $n$-butanoic acid from Nacalai Tesque, Inc. (Kyoto).

\subsection{Extraction and isolation}

The seeds $(2.05 \mathrm{~kg})$ of $A$. indica, separated manually from the dried fruits, were pulverized and were extracted with $n$-hexane (2 L) under reflux (3 h) three times which gave an $n$-hexane extract $(280 \mathrm{~g})$. The defatted residue was then extracted with $\mathrm{MeOH}(2 \mathrm{~L}$ ) at room temp ( $3 \mathrm{~d}$ ) three times. The $\mathrm{MeOH}$ extract $\left(255 \mathrm{~g}\right.$ ) was mixed with $\mathrm{H}_{2} \mathrm{O}$ and extracted with $\mathrm{CHCl}_{3}$ which afforded a $\mathrm{CHCl}_{3}$-soluble fraction $(73 \mathrm{~g})$. Column chromatography on silica gel $(750 \mathrm{~g})$ of the $\mathrm{CHCl}_{3}$ fraction $(72 \mathrm{~g}$ ), eluted with solvents of increasing polarity ( $n$-hexane-ethyl acetate $(\mathrm{EtOAc}), 9: 1 \rightarrow 0: 1)$, afforded 10 fractions, A-J, listed in increasing order of polarity. Fraction E (4.58 g) was passed through silica gel with $n^{-}$ hexane-EtOAc (3:2) and ODS with $\mathrm{MeOH}-\mathrm{H}_{2} \mathrm{O}(2: 3 \rightarrow 1: 0)$, and the fractions obtained were purified by preparative HPLC (HPLC system IV) to yield compounds $3(1.7 \mathrm{mg}), 6$ (1051 mg), 7 (4.6 mg), 10 (10.1 mg), 13 (2.2 mg), 14 (2.7 mg), $17(11.7 \mathrm{mg}), 18(2.3 \mathrm{mg})$, and 19 (4.0 mg). Fraction F (3421 $\mathrm{mg})$, on passing through silica gel with $n$-hexane-EtOAc $(4: 1 \rightarrow 0: 1)$, gave purified fraction $\mathrm{F}(1501 \mathrm{mg})$. Preparative HPLC (HPLC system I) of a portion $(390 \mathrm{mg}$ ) of this fraction afforded compounds 4 (6.9 mg), 8 ( $3.8 \mathrm{mg}), 9(5.2 \mathrm{mg})$, and 20 $(7.8 \mathrm{mg})$. A portion $(3051 \mathrm{mg})$ of fraction $\mathrm{G}(4442 \mathrm{mg})$ was chromatographed on silica gel with $n$-hexane-EtOAc (7:3 $\rightarrow 0: 1)$ to give 12 fractions, G1-G12. Subfractions G2 (283 mg), G3 (798 mg), G4 (419 mg), G6 (144 mg), and G7 (67 mg) were subjected to preparative HPLC (HPLC systems II, III, and IV) to afford compounds 1 (202.8 mg), 2 (7.9 mg), 4 (69.5 mg), 5 (1.8 mg), 8 (29.9 mg), 9 (14.7 mg), 12 (8.0 mg), 15 (1.6 $\mathrm{mg}), 20$ (26.2 mg), 26 (41.4 mg), $27(6.0 \mathrm{mg}), 29(1.2 \mathrm{mg})$ and $30(1.9 \mathrm{mg}), 31(2.3 \mathrm{mg})$, and $32(3.1 \mathrm{mg})$. Fraction I $(2251 \mathrm{mg})$ was passed through silica gel with $n$-hexane-EtOAc (2:3 $\rightarrow 0: 1$ ) and preparative HPLC (HPLC systems V and VI) of the fractions obtained gave compounds $11(5.9 \mathrm{mg}), 15(0.7$ mg), 21 (20.0 mg), 22 (3.7 mg), 23 (7.9 mg), 24 (2.9 mg), 25 (2.2 $\mathrm{mg})$, and 28 (5.3 mg).

\subsection{Identification and characterization}

Identification of the following 23 compounds was performed by ${ }^{1} \mathrm{H}$ NMR and MS comparison with the corresponding compounds in the literature: $1^{6)}, 2^{7}, 4^{8)}, 6^{9)}, 7^{7)}, 8^{8)}$, $11^{10)}, 15^{11)}, 16^{12)}, 17^{13)}, 18^{14)}, 19^{7)}, 20^{15)}, 21^{16)}, 22^{17)}, 23^{18)}, 25^{19)}$, $26^{20)}, 27^{21}, 28^{22)}, 29^{6)}, 30^{6}$, and $31^{6)}$ (For the names and structures of these compounds, refer to Table 3 and Fig. 1, respectively.). Six compounds, 3, 5, 12-14, and 32, are new compounds, and their characterization was performed by spectroscopic methods. The ${ }^{13} \mathrm{C}$ and ${ }^{1} \mathrm{H}$ NMR data for the new compounds are shown in Tables 1 and 2, respectively. Identification of compounds $9^{23)}$ and $24^{24)}$ was undertaken based on the NMR and mass spectral analysis because their spectral data were unavailable in the literature. Although compound 10 has previously been known as a synthetic compound $d^{9)}$, its isolation from a natural source seems to be the first instance in this study.

2.5.1 15-Hydroxyazadiradione (3)

Compound 3 was an amorphous solid; $[\alpha]^{25}+82.9(c 0.20$, $\mathrm{MeOH}) ; \mathrm{UV}(\mathrm{EtOH}) \lambda_{\max }(\log \varepsilon) 222$ (3.91), 270 (3.62) nm; IR 


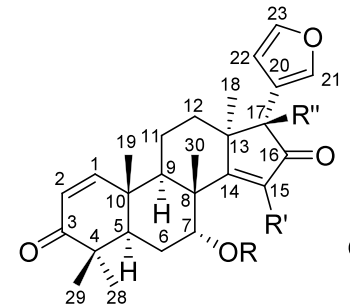

$1 \mathrm{R}=\mathrm{Ac} \mathrm{R}^{\prime}=\mathrm{R}^{\prime \prime}=\mathrm{H}$

$2 \mathrm{R}=\mathrm{Bz} \mathrm{R}^{\prime}=\mathrm{R}^{\prime \prime}=\mathrm{H}$

$3 \mathrm{R}=\mathrm{Ac} \mathrm{R}^{\prime}=\mathrm{OH} \mathrm{R}^{\prime \prime}=\mathrm{H}$

$4 \mathrm{R}=\mathrm{Ac} \quad \mathrm{R}^{\prime}=\mathrm{H} \quad \mathrm{R}^{\prime \prime}=\mathrm{OH}$

$5 \mathrm{R}=\mathrm{Bz} \mathrm{R}^{\prime}=\mathrm{H} \mathrm{R}^{\prime \prime}=\mathrm{OH}$<smiles>[3H]C1C=C2[C@@](C)(CC[C@@]3(C)[C@]2(C)[C@@H](OC(C)=O)C[C@@H]2C(C)(C)C(=O)C=C[C@]23C)[C@H]1C1=CCOC1=O</smiles>
$11 \mathrm{R}=\mathrm{O}$ $12 \mathrm{R}=\mathrm{H}_{2}$

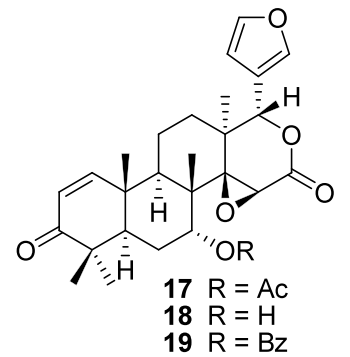

$19 \mathrm{R}=\mathrm{Bz}$

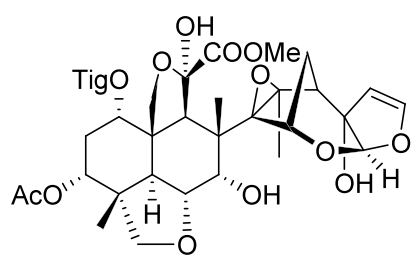

22

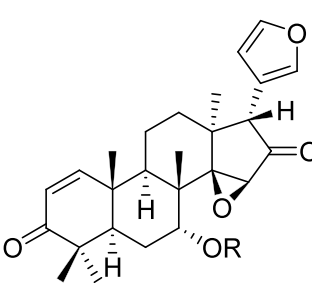

$\begin{array}{ll}6 & R=A c \\ 7 & R=B z\end{array}$

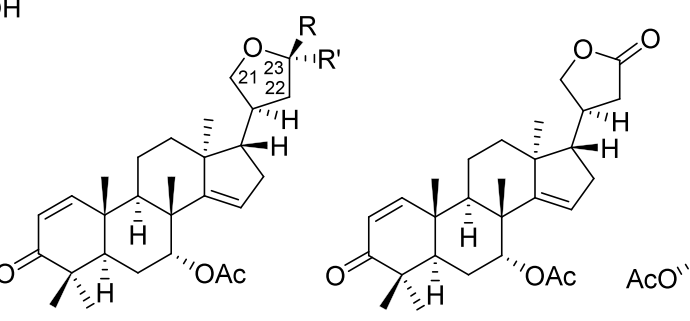

15

$13 \mathrm{R}=\mathrm{H} \mathrm{R}^{\prime}=\mathrm{OMe}$

$8 \mathrm{R}=\mathrm{H}$
$9 \mathrm{R}=\mathrm{OH}$

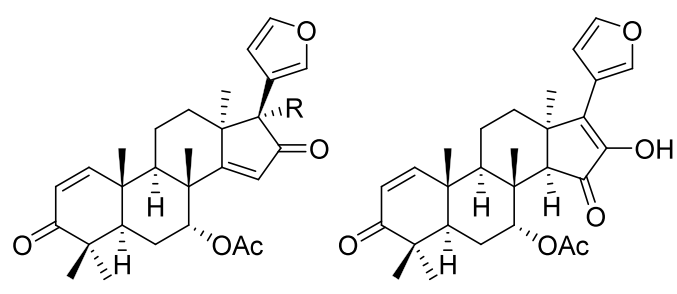

10<smiles>c1ccoc1</smiles>

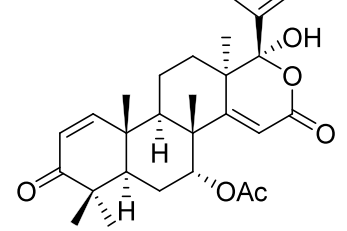

20

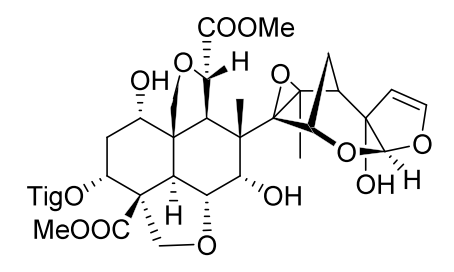

21

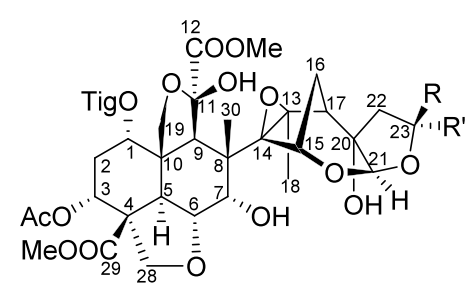

$23 \mathrm{R}=\mathrm{OMe} \mathrm{R}^{\prime}=\mathrm{H}$

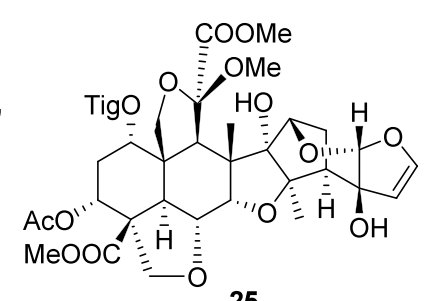

25

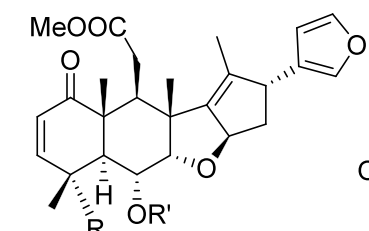

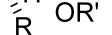

$26 \mathrm{R}=\mathrm{COOMe} \mathrm{R}^{\prime}=\mathrm{Ac}$

$27 \mathrm{R}=$ COOMe $\mathrm{R}^{\prime}=\mathrm{H}$

$28 \mathrm{R}=\mathrm{OH} \mathrm{R}^{\prime}=\mathrm{Ac}$

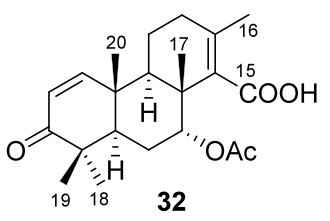

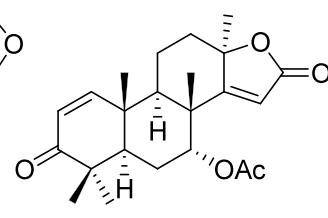

29<smiles>CC(=O)O[C@H]1C[C@H]2[C@@H](C)C(=O)C=C[C@]2(C)[C@@H]2CC[C@]3(C)OC(=O)C=C3[C@]12C</smiles>

30<smiles>CC(=O)O[C@H]1C[C@H]2[C@@H](C)C(=O)C=C[C@]2(C)[C@@H]2CC[C@H]3CC(=O)C=C3[C@H]12</smiles>

31<smiles>CC=CC(=O)C(C)=CCC=[Ge]</smiles>

Fig. 1 Structures of Compounds 1-32. 
T. Akihisa, T. Noto, A. Takahashi et al.

Table $1{ }^{13} \mathrm{C}$ NMR Spectroscopic Data ( $\delta$ values; $125 \mathrm{MHz}, \mathrm{CDCl}_{3}$ ) for Six Compounds from the Seeds of Azadirachta indica.

\begin{tabular}{|c|c|c|c|c|c|c|}
\hline C no. & $3^{\text {a) }}$ & 5 & $12^{\mathrm{a})}$ & 13 & 14 & 32 \\
\hline 1 & $156.9 \mathrm{~d}$ & $157.0 \mathrm{~d}$ & $158.1 \mathrm{~d}$ & $158.1 \mathrm{~d}$ & $158.1 \mathrm{~d}$ & $158.0 \mathrm{~d}$ \\
\hline 2 & $125.9 \mathrm{~d}$ & $125.9 \mathrm{~d}$ & $125.4 \mathrm{~d}$ & $125.5 \mathrm{~d}$ & $125.5 \mathrm{~d}$ & $125.9 \mathrm{~d}$ \\
\hline 3 & $204.3 \mathrm{~s}$ & $204.1 \mathrm{~s}$ & $204.5 \mathrm{~s}$ & $204.5 \mathrm{~s}$ & $204.6 \mathrm{~s}$ & $204.8 \mathrm{~s}$ \\
\hline 4 & $44.1 \mathrm{~s}$ & $44.1 \mathrm{~s}$ & $44.1 \mathrm{~s}$ & $44.1 \mathrm{~s}$ & $44.1 \mathrm{~s}$ & $44.0 \mathrm{~s}$ \\
\hline 5 & $46.2 \mathrm{~d}$ & $46.5 \mathrm{~d}$ & $46.2 \mathrm{~d}$ & $46.2 \mathrm{~d}$ & $46.2 \mathrm{~d}$ & $45.2 \mathrm{~d}$ \\
\hline 6 & $22.9 \mathrm{t}$ & $23.7 \mathrm{t}$ & $23.8 \mathrm{t}$ & $23.8 \mathrm{t}$ & $23.8 \mathrm{t}$ & $23.1 \mathrm{t}$ \\
\hline 7 & $74.4 \mathrm{~d}$ & $74.3 \mathrm{~d}$ & $74.5 \mathrm{~d}$ & $74.5 \mathrm{~d}$ & $74.5 \mathrm{~d}$ & $74.2 \mathrm{~d}$ \\
\hline 8 & $43.6 \mathrm{~s}$ & $45.0 \mathrm{~s}$ & $42.9 \mathrm{~s}$ & $42.7 \mathrm{~s}$ & $42.7 \mathrm{~s}$ & $42.1 \mathrm{~s}$ \\
\hline 9 & $38.9 \mathrm{~d}$ & $39.0 \mathrm{~d}$ & $38.4 \mathrm{~d}$ & $38.3 \mathrm{~d}$ & $38.6 \mathrm{~d}$ & $44.1 \mathrm{~d}$ \\
\hline 10 & $40.0 \mathrm{~s}$ & $40.2 \mathrm{~s}$ & $39.9 \mathrm{~s}$ & $39.9 \mathrm{~s}$ & $40.2 \mathrm{~s}$ & $39.2 \mathrm{~s}$ \\
\hline 11 & $16.1 \mathrm{t}$ & $15.7 \mathrm{t}$ & $16.4 \mathrm{t}$ & $16.4 \mathrm{t}$ & $16.5 \mathrm{t}$ & $17.4 \mathrm{t}$ \\
\hline 12 & $31.5 \mathrm{t}$ & $21.8 \mathrm{t}$ & $32.8 \mathrm{t}$ & $33.9 \mathrm{t}$ & $33.8 \mathrm{t}$ & $32.6 \mathrm{t}$ \\
\hline 13 & $43.7 \mathrm{~s}$ & $50.5 \mathrm{~s}$ & $47.3 \mathrm{~s}$ & $46.7 \mathrm{~s}$ & $46.6 \mathrm{~s}$ & $139.6 \mathrm{~s}$ \\
\hline 14 & $155.2 \mathrm{~s}$ & $193.1 \mathrm{~s}$ & $158.7 \mathrm{~s}$ & $158.7 \mathrm{~s}$ & $158.7 \mathrm{~s}$ & $133.4 \mathrm{~s}$ \\
\hline 15 & $144.2 \mathrm{~s}$ & $120.2 \mathrm{~d}$ & $118.5 \mathrm{~d}$ & $119.0 \mathrm{~d}$ & $119.1 \mathrm{~d}$ & $170.8 \mathrm{~s}$ \\
\hline 16 & $199.9 \mathrm{~s}$ & $206.0 \mathrm{~s}$ & $34.0 \mathrm{t}$ & $35.3 \mathrm{t}$ & $35.4 \mathrm{t}$ & $21.5 \mathrm{t}$ \\
\hline 17 & $57.4 \mathrm{~d}$ & $80.5 \mathrm{~s}$ & $50.8 \mathrm{~d}$ & $59.1 \mathrm{~d}$ & $58.3 \mathrm{~d}$ & $22.1 \mathrm{q}$ \\
\hline 18 & $27.5 \mathrm{q}$ & $30.5 \mathrm{q}$ & $20.9 \mathrm{q}$ & $20.2 \mathrm{q}$ & $19.8 \mathrm{q}$ & $27.3 \mathrm{q}$ \\
\hline 19 & $18.1 \mathrm{q}$ & $19.0 \mathrm{q}$ & $19.0 \mathrm{q}$ & $19.0 \mathrm{q}$ & $19.0 \mathrm{q}$ & $21.2 \mathrm{q}$ \\
\hline 20 & $118.4 \mathrm{~s}$ & $122.5 \mathrm{~s}$ & $134.2 \mathrm{~s}$ & $37.8 \mathrm{~d}$ & $40.2 \mathrm{~d}$ & $18.7 \mathrm{q}$ \\
\hline 21 & $141.4 \mathrm{~s}$ & $141.4 \mathrm{~d}$ & $174.2 \mathrm{~s}$ & $71.8 \mathrm{t}$ & $70.1 \mathrm{t}$ & \\
\hline 22 & $111.0 \mathrm{~d}$ & $109.5 \mathrm{~d}$ & $146.4 \mathrm{~d}$ & $39.0 \mathrm{t}$ & $38.6 \mathrm{t}$ & \\
\hline 23 & $143.0 \mathrm{~d}$ & $142.7 \mathrm{~d}$ & $70.2 \mathrm{t}$ & $104.3 \mathrm{~d}$ & $105.1 \mathrm{~d}$ & \\
\hline 28 & $27.0 \mathrm{q}$ & $27.1 \mathrm{q}$ & $27.0 \mathrm{q}$ & $27.0 \mathrm{q}$ & $27.0 \mathrm{q}$ & \\
\hline 29 & $21.3 \mathrm{q}$ & $21.2 \mathrm{q}$ & $21.3 \mathrm{q}$ & $21.3 \mathrm{q}$ & $21.3 \mathrm{q}$ & \\
\hline 30 & $21.5 \mathrm{q}$ & $25.5 \mathrm{q}$ & $27.4 \mathrm{q}$ & $27.3 \mathrm{q}$ & $27.3 \mathrm{q}$ & \\
\hline OCEOMe & $169.8 \mathrm{~s}$ & & $170.0 \mathrm{~s}$ & $170.1 \mathrm{~s}$ & $170.1 \mathrm{~s}$ & $171.6 \mathrm{~s}$ \\
\hline $\mathrm{OCOMe}$ & $21.1 \mathrm{q}$ & & $21.2 \mathrm{q}$ & $21.1 \mathrm{q}$ & $21.3 \mathrm{q}$ & $21.0 \mathrm{q}$ \\
\hline $\mathrm{OMe}$ & & & & $54.6 \mathrm{q}$ & $55.1 \mathrm{q}$ & \\
\hline $\mathrm{OC} \underline{\mathrm{COPh}}$ & & $165.2 \mathrm{~s}$ & & & & \\
\hline $1^{\prime}$ & & $129.7 \mathrm{~s}$ & & & & \\
\hline $2^{\prime}$ & & $129.4 \mathrm{~d}$ & & & & \\
\hline $3^{\prime}$ & & $128.5 \mathrm{~d}$ & & & & \\
\hline $4^{\prime}$ & & $133.3 \mathrm{~d}$ & & & & \\
\hline $5^{\prime}$ & & $128.5 \mathrm{~d}$ & & & & \\
\hline $6^{\prime}$ & & $129.4 \mathrm{~d}$ & & & & \\
\hline
\end{tabular}

a) Determined at $150 \mathrm{MHz}$. 
Table $2{ }^{1} \mathrm{H} \mathrm{NMR}^{1}$ Spectroscopic Data ( $\delta$ values; $500 \mathrm{MHz}, \mathrm{CDCl}_{3}$ ) for Six Compounds from the Seeds of Azadirachta indica ${ }^{\text {a) }}$

\begin{tabular}{|c|c|c|c|c|c|c|}
\hline H no & $3^{\mathrm{b})}$ & 5 & $12^{\mathrm{b})}$ & 13 & 14 & 32 \\
\hline 1 & $7.13(\mathrm{~d}, 10.4)$ & $7.21(\mathrm{~d}, 10.3)$ & $7.16(\mathrm{~d}, 10.3)$ & $7.15(\mathrm{~d}, 10.1)$ & $7.14(\mathrm{~d}, 10.3)$ & $7.18(\mathrm{~d}, 10.3)$ \\
\hline 2 & $5.88(\mathrm{~d}, 10.4)$ & $5.91(\mathrm{~d}, 10.3)$ & $5.85(\mathrm{~d}, 10.3)$ & $5.85(\mathrm{~d}, 10.1)$ & $5.85(\mathrm{~d}, 10.3)$ & $5.89(\mathrm{~d}, 10.3)$ \\
\hline 5 & $2.18(\mathrm{dd}, 2.6,12.9)$ & $2.33(\mathrm{dd}, 2.6,12.9)$ & 2.18 & $2.19(\mathrm{dd}, 2.5,13.1)$ & $2.18(\mathrm{dd}, 3.4,14.4)$ & 2.11 \\
\hline 6 & $2.06(\alpha)$ & $2.04(\alpha)$ & $1.79(\alpha)$ & $1.78(\alpha, \mathrm{dt}, 14.6,2.5)$ & $1.78(\alpha, \mathrm{dt}, 14.6,2.9)$ & $1.88(\alpha, \mathrm{dd}, 2.9,9.7)$ \\
\hline & $1.95(\beta)$ & $2.13(\beta)$ & $1.95(\beta)$ & $1.95(\beta)$ & $1.93(\beta)$ & $1.88(\beta, \mathrm{dd}, 2.9,9.7)$ \\
\hline 7 & $5.62(\mathrm{t}, 3.1)$ & $5.63(\mathrm{t}, 2.8)$ & $5.27(\mathrm{t}, 2.8)$ & $5.23(\mathrm{t}, 2.5)$ & $5.24(\mathrm{t}, 2.5)$ & $5.28(\mathrm{t}, 2.9)$ \\
\hline 9 & 2.52 & $2.53(\mathrm{dd}, 6.8,11.7)$ & 2.20 & $2.22(\mathrm{dd}, 6.7,12.2)$ & 2.21 & 1.79 \\
\hline 11 & $2.09(\alpha)$ & $2.15(\alpha)$ & $1.93(\alpha)$ & $1.97(\alpha)$ & $1.95(\alpha)$ & $1.92(\alpha)$ \\
\hline & $1.87(\beta)$ & $1.98(\beta)$ & $1.75(\beta)$ & $1.70(\beta)$ & $1.69(\beta)$ & $1.79(\beta)$ \\
\hline 12 & $2.15(\alpha)$ & $1.64(\alpha$, ddd $, 2.7,9.9,12.9)$ & $2.04(\alpha)$ & $1.72(\alpha)$ & $1.72(\alpha)$ & $2.27(\alpha)$ \\
\hline- & $1.85(\beta)$ & $2.59(\beta)$ & $1.77(\beta)$ & $1.52(\beta)$ & $1.54(\beta)$ & $2.27(\beta)$ \\
\hline 15 & & $5.83(\mathrm{~s})$ & $5.36(\mathrm{dd}, 1.4,3.4)$ & $5.28(\mathrm{dd}, 1.7,3.5)$ & $5.28(\mathrm{dd}, 1.7,3.5)$ & \\
\hline 16 & & & $2.51(\alpha$, ddd, $1.4,11.2,15.3)$ & $2.03(\alpha)$ & $2.03(\alpha)$ & $1.83(\mathrm{~s})$ \\
\hline 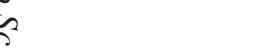 & & & $2.30(\beta, \mathrm{ddd}, 3.4,7.2,15.3)$ & $2.14(\beta$, ddd $, 3.5,7.5,15.7)$ & $2.15(\beta, \mathrm{dd}, 3.5,7.2)$ & \\
\hline 17 & $3.37(\mathrm{~s})$ & & 2.84 (br. t, 8.8) & 1.57 & $1.64(\mathrm{dt}, 7.4,10.6)$ & $1.39(\mathrm{~s})$ \\
\hline 18 & $1.04(\mathrm{~s})$ & $0.95(\mathrm{~s})$ & $0.86(\mathrm{~s})$ & $1.01(\mathrm{~s})$ & $0.98(\mathrm{~s})$ & $1.05(\mathrm{~s})$ \\
\hline 19 & $1.25(\mathrm{~s})$ & $1.31(\mathrm{~s})$ & $1.20(\mathrm{~s})$ & $1.17(\mathrm{~s})$ & $1.17(\mathrm{~s})$ & $1.08(\mathrm{~s})$ \\
\hline 20 & & & & 2.56 & 2.26 & $1.13(\mathrm{~s})$ \\
\hline 21 & 7.45 (br. s) & 7.49 (br. s) & & $4.11[\alpha($ pro $-S), \mathrm{t}, 8.3]$ & $3.96[\alpha($ pro- $S), \mathrm{t}, 7.8]$ & \\
\hline & & & & $3.48[\beta($ pro $-R), \mathrm{t}, 8.3]$ & $3.44[\mathrm{~b}($ pro- $R), \mathrm{t}, 8.3]$ & \\
\hline 22 & $6.25(\mathrm{t}-\mathrm{like}, 1.2)$ & 6.35 (br. s) & $7.22(\mathrm{dd}, 1.7,3.1)$ & $1.97[\alpha($ pro $-R)]$ & $2.24[\alpha($ pro- $R)]$ & \\
\hline & & & & $1.54[\beta($ pro- $S)]$ & $1.49[\beta($ pro-S $)]$ & \\
\hline 23 & 7.44 (t-like, 1.2) & 7.39 & $4.82(\mathrm{dt}, 18.0,1.0)$ & $4.92(\mathrm{~d}, 4.9)$ & $4.99(\mathrm{dd}, 3.7,5.2)$ & \\
\hline & & & $4.86(\mathrm{dt}, 18.0,1.0)$ & & & \\
\hline 28 & $1.09(\mathrm{~s})$ & $1.00(\mathrm{~s})$ & $1.08(\mathrm{~s})$ & $1.07(\mathrm{~s})$ & $1.07(\mathrm{~s})$ & \\
\hline 29 & $1.10(\mathrm{~s})$ & $1.11(\mathrm{~s})$ & $1.08(\mathrm{~s})$ & $1.07(\mathrm{~s})$ & $1.07(\mathrm{~s})$ & \\
\hline 30 & $1.36(\mathrm{~s})$ & $1.44(\mathrm{~s})$ & $1.23(\mathrm{~s})$ & $1.16(\mathrm{~s})$ & $1.16(\mathrm{~s})$ & \\
\hline OCOMe & $1.95(\mathrm{~s})$ & & $1.96(\mathrm{~s})$ & $1.94(\mathrm{~s})$ & $1.94(\mathrm{~s})$ & $2.00(\mathrm{~s})$ \\
\hline $\mathrm{OMe}$ & & & & $3.33(\mathrm{~s})$ & $3.35(\mathrm{~s})$ & \\
\hline $2^{\prime}$ & & $7.89(\mathrm{dd}, 1.4,8.6)$ & & & & \\
\hline $3^{\prime}$ & & 7.38 & & & & \\
\hline $4^{\prime}$ & & $7.53(\mathrm{tt}, 1.4,7.4)$ & & & & \\
\hline $5^{\prime}$ & & 7.38 & & & & \\
\hline $6^{\prime}$ & & $7.89(\mathrm{dd}, 1.4,8.6)$ & & & & \\
\hline
\end{tabular}

a) $J$ values $(\mathrm{Hz})$ determined are shown in parentheses.

b) Determined at $600 \mathrm{MHz}$. 


\section{T. Akihisa, T. Noto, A. Takahashi et al.}

$(\mathrm{KBr}) \mathrm{v}_{\max } 3423(\mathrm{OH}), 1736$ (ester $\left.\mathrm{C}=\mathrm{O}\right), 1719$ (conjugated cyclopentenone), 1669 (conjugated cyclohexenone), 870 (furan), $826(>\mathrm{C}=\mathrm{CH}-) \mathrm{cm}^{-1},{ }^{13} \mathrm{C}$ and ${ }^{1} \mathrm{H}$ NMR, see Tables 1 and 2, respectively; HR-ESIMS $m / z 489.2269$ (calculated for $\mathrm{C}_{28} \mathrm{H}_{34} \mathrm{O}_{6} \mathrm{Na}[\mathrm{M}+\mathrm{Na}]^{+}$, 489.2253).

2.5.2 7-Benzoyl-17-hydroxynimbocinol (5)

Compound 5 was an amorphous solid; $[a]_{\mathrm{D}}^{25}+40.3$ (c 0.12 , EtOH); UV (EtOH) $\lambda_{\max }(\log \varepsilon) 231$ (4.31) nm; IR (KBr) $\mathrm{v}_{\max }$ $3447(\mathrm{OH}), 1730$ (ester $\mathrm{C}=\mathrm{O}), 1714$ (conjugated cyclopentenone), 1672 (conjugated cyclohexenone), 874 (furan), 820 $(>\mathrm{C}=\mathrm{CH}-) \mathrm{cm}^{-1} ;{ }^{13} \mathrm{C}$ and ${ }^{1} \mathrm{H}$ NMR, see Tables 1 and 2 , respectively; HR-ESIMS $m / z 551.2410$ (calculated for $\mathrm{C}_{33} \mathrm{H}_{36} \mathrm{O}_{6} \mathrm{Na}[\mathrm{M}+\mathrm{Na}]^{+}$, 551.2404).

2.5.3 17-Epi-17-hydroxyazadiradione (9)

${ }^{13} \mathrm{C}(150 \mathrm{MHz})$ and ${ }^{1} \mathrm{H}$ NMR $(600 \mathrm{MHz}): \mathrm{C}-1\left[\delta_{\mathrm{C}} 157.1 ; \delta_{\mathrm{H}}\right.$ $7.05(\mathrm{~d}, J=10.0 \mathrm{~Hz})], \mathrm{C}-2$ [125.8; $5.88(\mathrm{~d}, 10.0)], \mathrm{C} 3$ [204.3], C-4 [44.0], C-5 [45.6; 2.16 (dd, 3.5, 10.7)], C-6 [22.6; $1.92(2 \mathrm{H})]$, C-7 [74.3; 5.29 (t, 2.7)], C-8 [42.0], C-9 [42.1; 1.93 (dd, 3.1, 10.3)], C-10 [40.7], C-11 [17.9; $2.13(\alpha), 1.85(\beta)], \mathrm{C}-12$ [30.4; $2.16(\alpha), 1.86(\beta)], \mathrm{C}-13$ [40.6], C-14 [57.1; $2.55(\mathrm{~s})], \mathrm{C}-15$ [200.4], C-16 [146.9], C-17 [140.9], C-18 [27.7; $1.44(\mathrm{~s})], \mathrm{C}-19$ [18.2; 1.21 (s)], C-20 [117.9], C-21 [144.1; $8.02(\mathrm{~s})]$, C-22 [109.9; $6.92(\mathrm{dd}, 0.7,2.1)], \mathrm{C}-23$ [143.2; 7.51 (t, 1.7)], C-28 [27.1; 1.09 (s)], C-29 [21.4; $1.08(\mathrm{~s})], \mathrm{C}-30$ [18.8; $0.94(\mathrm{~s})], 16-\mathrm{OH}$ [5.80 (s)], 7-OCOMe [169.3], 7-OCOMe [21.2; 2.07 (s)]; ESIMS $m / z$ $467[\mathrm{M}+\mathrm{H}]^{+}$.

2.5.4 7-Acetyl-16,17-dehydro-16-hydroxyneotrichi lenone (10)

${ }^{13} \mathrm{C}(150 \mathrm{MHz})$ and ${ }^{1} \mathrm{H}$ NMR $(600 \mathrm{MHz}): \mathrm{C}-1\left[\delta_{\mathrm{C}} 157.1 ; \delta_{\mathrm{H}}\right.$ $7.05(\mathrm{~d}, J=10.0 \mathrm{~Hz})], \mathrm{C}-2$ [125.8; $5.88(\mathrm{~d}, 10.0)], \mathrm{C}-3$ [204.3], C-4 [44.0], C-5 [45.6; 2.16 (dd, 3.5, 10.7)], C-6 [22.6; $1.92(2 \mathrm{H})]$, C-7 [74.3; 5.29 (t, 2.7)], C-8 [42.0], C-9 [42.1; 1.93 (dd, 3.1, 10.3)], C-10 [40.7], C-11 [17.9; $2.13(\alpha), 1.85(\beta)], \mathrm{C}-12$ [30.4; $2.16(\alpha), 1.86(\beta)], \mathrm{C}-13$ [40.6], C-14 [57.1; $2.55(\mathrm{~s})], \mathrm{C}-15$ [200.4], C-16 [146.9], C-17 [140.9], C-18 [27.7; 1.44 (s)], C-19 [18.2; $1.21(\mathrm{~s})]$, C-20 [117.9], C-21 [144.1; 8.02 (s)], C-22 [109.9; $6.92(\mathrm{dd}, 0.7,2.1)], \mathrm{C}-23$ [143.2; 7.51 (t, 1.7)], C-28 [27.1; 1.09 (s)], C-29 [21.4; $1.08(\mathrm{~s})], \mathrm{C}-30$ [18.8; $0.94(\mathrm{~s})], 16-\mathrm{OH}[5.80(\mathrm{~s})]$, 7-OCOMe [169.3], 7-OCOMe [21.2; 2.07 (s)]; ESIMS $\mathrm{m} / z$ $467[\mathrm{M}+\mathrm{H}]^{+}$.

2.5.5 3-Dehydroxyazadironolide (12)

Compound 12 was an amorphous solid; $[\alpha]_{\mathrm{D}}^{25}+49.6$ (c 0.25 , $\left.\mathrm{CHCl}_{3}\right)$; UV (EtOH) $\lambda_{\max }(\log \varepsilon) 210$ (4.16) nm; IR (KBr) $\mathrm{v}_{\max }$ 1745 ( $\alpha, \beta$-unsaturated $\gamma$-lactone), 1728 (ester $\mathrm{C}=\mathrm{O}), 1670$ (conjugated cyclohexenone), $827(>\mathrm{C}=\mathrm{CH}-) \mathrm{cm}^{-1} ;{ }^{13} \mathrm{C}$ and ${ }^{1} \mathrm{H}$ NMR, see Tables 1 and 2, respectively; HR-ESIMS $m / z$ 453.2629 (calculated for $\mathrm{C}_{28} \mathrm{H}_{37} \mathrm{O}_{5}[\mathrm{M}+\mathrm{H}]^{+}$, 453.2640).

2.5.6 Limocin E (13)

Compound 13 was an amorphous solid; $[\alpha]_{\mathrm{D}}^{25}+33.5(c 0.18$, EtOH); UV (EtOH) $\lambda_{\max }(\log \varepsilon) 226(4.03) \mathrm{nm}$; IR (KBr) $\mathrm{v}_{\max }$ 1738 (ester $\mathrm{C}=\mathrm{O}), 1672$ (conjugated cyclohexenone), 822 $(>\mathrm{C}=\mathrm{CH}-) \mathrm{cm}^{-1} ;{ }^{13} \mathrm{C}$ and ${ }^{1} \mathrm{H} \mathrm{NMR}$, see Tables 1 and 2 , respectively; HR-ESIMS $m / z 471.3100$ (calculated for $\mathrm{C}_{29} \mathrm{H}_{43} \mathrm{O}_{5}[\mathrm{M}+\mathrm{H}]^{+}$, 471.3115).
2.5.7 23-Epilimocin E (14)

Compound 14 was an amorphous solid; $[\alpha]_{\mathrm{D}}^{25}-43.6$ (c 0.20, $\mathrm{EtOH})$ UV (EtOH) $\lambda_{\max }(\log \varepsilon) 228(4.01) \mathrm{nm}$; IR $(\mathrm{KBr}) \mathrm{v}_{\max }$ 1738 (ester $\mathrm{C}=\mathrm{O}$ ), 1672 (conjugated cyclohexenone) $\mathrm{cm}^{-1}$; ${ }^{13} \mathrm{C}$ and ${ }^{1} \mathrm{H}$ NMR, see Tables 1 and 2, respectively; HRESIMS $m / z 471.3093$ (calculated for $\mathrm{C}_{29} \mathrm{H}_{43} \mathrm{O}_{5}[\mathrm{M}+\mathrm{H}]^{+}$, 471.3105).

2.5.8 23-Epivepaol (24)

${ }^{13} \mathrm{C}(125 \mathrm{MHz})$ and ${ }^{1} \mathrm{H}$ NMR $(500 \mathrm{MHz})$ : $\mathrm{C}-1\left[\delta_{\mathrm{C}} 70.6 ; \delta_{\mathrm{H}} 4.77\right.$ $(\mathrm{t}, J=2.9 \mathrm{~Hz})], \mathrm{C}-2[29.8 ; 2.23(\alpha, \mathrm{dt}, 3.3,16.9), 2.34(\beta)], \mathrm{C}-3$ [67.0; $5.51(\mathrm{t}, 2.9)], \mathrm{C}-4$ [125.3], C-5 [37.1; 3.38 (d, 12.6)], C-6 [73.9; $4.60(\mathrm{dd}, 2.6,12.6)], \mathrm{C}-7$ [74.2; $4.71(\mathrm{~d}, 2.6)], \mathrm{C}-8$ [52.5], C-9 [44.8; $3.32(\mathrm{~s})]$, C-10 [50.2], C-11 [104.2], C-12 [171.8], C13 [68.4], C-14 [69.5], C-15 [77.2; 4.69 (d, 3.2)], C-16 [24.5; $1.43(\alpha, \mathrm{d}, 12.9), 1.69(\beta)], \mathrm{C}-17$ [49.0; $2.45(\mathrm{~d}, 5.5)], \mathrm{C}-18$ [18.4; $2.00(\mathrm{~s})$ ], C-19 [69.1; $3.62(\alpha, \mathrm{d}, 9.7), 4.15(\beta, \mathrm{d}, 9.7)], \mathrm{C}-20$ [80.0], C-21 [106.7; $5.54(\mathrm{~s})], \mathrm{C}-22$ [45.6; $2.07(\alpha, \mathrm{d}, 14.3), 2.32$ $(\beta)$ ], C-23 [104.0; $5.14(\mathrm{t}, 4.9)], \mathrm{C}-28$ [73.1; $3.76(\alpha, \mathrm{d}, 8.9), 4.07$ $(\beta, \mathrm{d}, 9.1)], \mathrm{C}-29$ [173.4], C-30 [21.4; 1.75 (s)], 3-OCOMe [169.6], 3-OCOMe [20.8; $1.93(\mathrm{~s})], 12-\mathrm{COOMe}$ [53.3; $3.68(\mathrm{~s})]$, 23-OMe [55.0; $3.39(\mathrm{~s})], 29-\mathrm{COOMe}[52.7 ; 3.79(\mathrm{~s})], \mathrm{C}-1^{\prime}$ [166.2], C-2' [128.6], C-3' [137.7; 6.94 (dq-like)], C-4' [14.3; 1.77 (d, 6.0)], C-5' [11.9; 1.85 (s)]; HR-ESIMS $m / z$ 775.2768 (calculated for $\mathrm{C}_{36} \mathrm{H}_{48} \mathrm{O}_{17} \mathrm{Na}[\mathrm{M}+\mathrm{Na}]^{+}$, 775.2783). 2.5.9 7 $\alpha$-Acetoxy-3-oxoisocopala-1,13-dien-15-oic acid (32)

Compound 32 was an amorphous solid; $[\alpha]_{\mathrm{D}}^{25}-31.7$ (c 0.19, $\mathrm{EtOH}) ; \mathrm{UV}(\mathrm{EtOH}) \lambda_{\max }(\log \varepsilon) 224(4.06) \mathrm{nm}$; IR $(\mathrm{KBr}) \mathrm{v}_{\max }$ 1739 (ester $\mathrm{C}=\mathrm{O}), 1723$ (carboxyl), 1671 (cyclohexenone) $\mathrm{cm}^{-}$ ${ }^{1}$; ${ }^{13} \mathrm{C}$ and ${ }^{1} \mathrm{H}$ NMR, see Tables 1 and 2, respectively; HRESIMS $m / z 397.1989$ (calculated for $\mathrm{C}_{22} \mathrm{H}_{30} \mathrm{O}_{5} \mathrm{Na}[\mathrm{M}+\mathrm{Na}]^{+}$, 397.1985).

\subsection{Cell culture}

B16 murine melanoma cells purchased from Riken Cell Bank (Tsukuba, Japan) were cultured in MEM supplemented with 5\% FBS, 100 units $/ \mathrm{mL}$ penicillin, and $100 \mu \mathrm{g} / \mathrm{mL}$ streptomycin at $37{ }^{\circ} \mathrm{C}$ in a humidified $\mathrm{CO}_{2}$ controlled (5\%) incubator.

\subsection{Determination of cell proliferation}

Cell proliferation was assessed using the MTT based colorimetric assay. After $72 \mathrm{~h}$ incubation with test compounds, MTT $(100 \mu \mathrm{L}, 5 \mathrm{mg} / \mathrm{mL}$ in phosphate-buffered saline) solution was added to the wells. After $3 \mathrm{~h}$ of incubation, the medium was removed, and 2-propanol containing $0.04 \mathrm{M} \mathrm{HCl}$ was added to dissolve the formazan produced in the cells. The absorbance of each well was then read at $570 \mathrm{~nm}$ (reference, $620 \mathrm{~nm}$ ) by using microplate reader (Labsystem Multiskan MS-UV). The optical density of formazan formed by control cells was used as a reference (assumed to be 100\%).

\subsection{Assay of melanin content}

Test samples dissolved in dimethylsulfoxide (DMSO) were 
added to the cell culture at final concentration of 25 $\mu \mathrm{g} / \mathrm{mL}$. B16 cells, plated at $2 \times 10^{5}$ cells in a culture dish (60 $\mathrm{mm}$ i.d.), were pre-incubated for $24 \mathrm{~h}$. After then, the medium was transferred into $5 \mathrm{~mL}$ of fresh medium containing the test sample and cultured for $3 \mathrm{~d}$. The cells were harvested by trypsinization. The samples were dissolved in $1 \mathrm{~mL}$ of $1 \mathrm{M} \mathrm{NaOH}$ containing 10\% DMSO, and the amount of melanin was determined spectrophotometrically by absorbance at $420 \mathrm{~nm}$ using microplate reader. The optical density of control cells was assumed to be $100 \%$.

\subsection{Assay of TPA-induced inflammation ear edema in mice}

For the protocol for this in vivo assay, refer to previous $\operatorname{article~}^{25)}$.

\subsection{In vitro EBV-EA activation experiment}

For the protocol for this in vitro assay, refer to previous $\operatorname{article~}^{25)}$.

\subsection{In vivo two-stage mouse skin carcinogenesis assay initiated by PN}

For the protocol for this in vivo assay, refer to previous $\operatorname{articles}^{26,27)}$.

\section{RESULTS AND DISCUSSION}

Twenty-eight limonoids, including 15 azadirone-type (1-15), one vilasinin-type (16), 4 gedunin-type (17-20), 5 azadirachtin-type (21-25), and 3 nimbin-type (26-28) limonoids, 3 degraded limonoids (29-31), and one diterpenoid (32), were isolated from a $\mathrm{CHCl}_{3}$-soluble fraction of a $\mathrm{MeOH}$ extract of the defatted seeds of Azadirachta indica (Fig. 1). Among these, six compounds, 3, 5, 12-14, and 32, are new compounds.

The molecular formula of compound 3 was determined to be $\mathrm{C}_{28} \mathrm{H}_{34} \mathrm{O}_{6}$ on the basis of its HR-ESIMS ( $[\mathrm{M}+\mathrm{Na}]^{+}, m / z$ 489.2269). The UV spectrum showed absorption maximum at $222 \mathrm{~nm}$ consistent with an $\alpha, \beta$-unsaturated ketone system while IR spectrum indicated the presence of hydroxyl $\left(3423 \mathrm{~cm}^{-1}\right)$, ester carbonyl $\left(1736 \mathrm{~cm}^{-1}\right)$, conjugated cyclopentenone $\left(1719 \mathrm{~cm}^{-1}\right)$, conjugated cyclohexenone $\left(1669 \mathrm{~cm}^{-1}\right)$, and furan ring $\left(870 \mathrm{~cm}^{-1}\right)$ groups. The ${ }^{13} \mathrm{C}$ NMR spectrum (Table 1) indicated the presence of 6 methyls, 3 methylenes, $4 s p^{3}$ methines of which one was an oxymethine $\left(\delta_{\mathrm{C}} 74.4\right), 3$ carbonyls including 2 ketones, 4 double bonds of which 2 are due to a furan ring, and $4 s p^{3}$ quaternary carbons. The ${ }^{1} \mathrm{H}$ NMR spectrum showed the presence of 5 tertiary methyls $\left(\delta_{\mathrm{H}} 1.04,1.09,1.10,1.25\right.$, and 1.36), one acetate methyl $\left(\boldsymbol{\delta}_{\mathrm{H}} 1.95\right)$, one oxymethine $\left(\boldsymbol{\delta}_{\mathrm{H}} 5.62\right)$, and a $\beta$ substituted furan ring $\left(\delta_{\mathrm{H}} 6.25,7.44 \text {, and } 7.45,1 \mathrm{H} \text { each }\right)^{6}$. The ${ }^{13} \mathrm{C}$ NMR signals of C-1 $\left(\delta_{\mathrm{C}} 156.9\right), \mathrm{C}-2\left(\delta_{\mathrm{C}} 125.9\right)$, and C$3\left(\boldsymbol{\delta}_{\mathrm{C}} 204.3\right)$ and the ${ }^{1} \mathrm{H}$ NMR signals of a pair of $\mathrm{AB}$ doublets at $\delta_{\mathrm{H}} 5.88$ and $7.13(\mathrm{~J}=10.4 \mathrm{~Hz})$ suggested that the A-ring of 3 possesses a 1-en-3-one system ${ }^{6}$. In the HMBC spectrum, the cross-correlation between $\delta_{\mathrm{H}} 5.62(\mathrm{H}-7)$ and $\delta_{\mathrm{C}}$ 169.8 demonstrated that the acetoxyl group is attached to C-7. In addition, diagnostic HMBC cross-correlations for H-17 (with C-12, C-13, C-16, C-20, C-21, and C-22) suggested the presence of a cyclopentenone group in the $\mathrm{D}$-ring. The ${ }^{13} \mathrm{C}$ and ${ }^{1} \mathrm{H}$ NMR spectra of 3 were very similar to those of azadiradione $(1)^{6,28)}$, except for the absence of a methine signal due to $\mathrm{H}-15$, suggesting that 3 was a $\mathrm{C}-15$ hydroxyl derivative of 1 . This was supported by the significant down-field shift of $\alpha$-carbon (C-15) $\left(\delta_{\mathrm{C}} 144.2\right.$ for 3 vs. $\delta_{\mathrm{C}}$ 123.5 for $\left.1^{6}\right)$ and up-field shift of $\beta$-carbon $(\mathrm{C}-14)\left(\delta_{\mathrm{C}} 155.2\right.$ for 3 vs. $\delta_{\mathrm{C}} 192.2$ for $1^{6}$ ) signals in the vinyl alcohol system of $3^{29)}$. The above evidence, coupled with the analysis of ${ }^{1} \mathrm{H}-{ }^{1} \mathrm{H}$ COSY, HMQC, and $\mathrm{HMBC}$ spectra, indicated that 3 possesses a $7 \alpha$-acetoxy-21,23-epoxy-15-hydroxy24,25,26,27-tetranorapotirucalla-1,14,20,22-tetraene-3,16dione (15-hydroxyazadiradione). The relative configuration was deduced from NOESY (Fig. 2) and molecular modeling $^{30)}$.

Compound 5 gave a $[\mathrm{M}+\mathrm{Na}]^{+}$ion in the HR-ESIMS at $m / z 551.2410$, consistent with molecular formula $\mathrm{C}_{33} \mathrm{H}_{36} \mathrm{O}_{6}$. Its IR spectrum exhibited absorption bands for hydroxyl, ester carbonyl, conjugated cyclopentenone, conjugated cyclohexenone, and furan ring groups. The ${ }^{13} \mathrm{C}$ NMR spectrum (Table 1) indicated the presence of 5 methyls, 3 methylenes, $3 s p^{3}$ methines of which one was an oxymethine $\left(\boldsymbol{\delta}_{\mathrm{C}} 74.3\right), 3$ carbonyls including 2 ketones, 7 double bonds of which 5 are due to a phenyl and a furan rings, and $5 s p^{3}$ quaternary carbons of which one was an oxygen bearing carbon $\left(\boldsymbol{\delta}_{\mathrm{C}} 80.5\right)$. The ${ }^{1} \mathrm{H}$ NMR spectrum of 5 was very similar to that of 7-benzoylnimbocinol $(2)^{7}$, except for the absence of a methine signal arising from $\mathrm{H}-17$, which suggested that 5 was a 17 -hydroxyl derivative of 2 . Thus, the structure of 5 was $7 \alpha$-benzoyloxy-21,23-epoxy-17 $\beta$ hydroxy-24,25,26,27-tetranorapotirucalla-1,14,20,22tetraene-3,16-dione (7-benzoyl-17-hydroxynimbocinol) which was supported from the analysis of ${ }^{1} \mathrm{H}-{ }^{1} \mathrm{H}$ COSY, HMQC, HMBC, and NOESY (Fig. 2) spectra.

The molecular formula of compound 12 was determined to be $\mathrm{C}_{28} \mathrm{H}_{36} \mathrm{O}_{5}$ on the basis of its HR-ESIMS $\left([\mathrm{M}+\mathrm{H}]^{+}, \mathrm{m} / z\right.$ 453.2629). The IR spectrum of 12 showed the absorption bands for ester carbonyl, conjugated cyclohexenone, and trisubstituted double bond groups. The ${ }^{13} \mathrm{C}$ (Table 1 ) and ${ }^{1} \mathrm{H}$ NMR spectra (Table 2) exhibited the presence of 5 tertiary methyls $\left[\delta_{\mathrm{H}} 0.86,1.08(2 \times \mathrm{xe}), 1.20\right.$, and 1.23$]$, one acetate methyl $\left(\delta_{\mathrm{H}} 1.96\right)$, one oxymethine $\left(\boldsymbol{\delta}_{\mathrm{C}} 74.5, \boldsymbol{\delta}_{\mathrm{H}} 5.27\right)$, one vinyl methine $\left(\delta_{\mathrm{C}} 118.5, \delta_{\mathrm{H}} 5.36\right)$, and the ring-A 1-en-3-one system $\left[\mathrm{AB}\right.$ doublets at $\delta_{\mathrm{H}} 7.16(\mathrm{H}-1)$ and $5.85(\mathrm{H}-2)(\boldsymbol{J}=10.3$ $\mathrm{Hz}) ; \delta_{\mathrm{C}} 158.1(\mathrm{C}-1), 125.4(\mathrm{C}-2)$, and $\left.204.5(\mathrm{C}-3)\right]^{6}$, suggesting that 12 possesses the tetracyclic skeleton and substituents identical to those of azadirone ( $7 \alpha$-acetoxy-21,23-epoxy24,25,26,27-tetranorapotirucalla-1,14,20,22-tetraen-3-one $)^{9)}$. The IR spectrum of 12 further showed absorption band at 

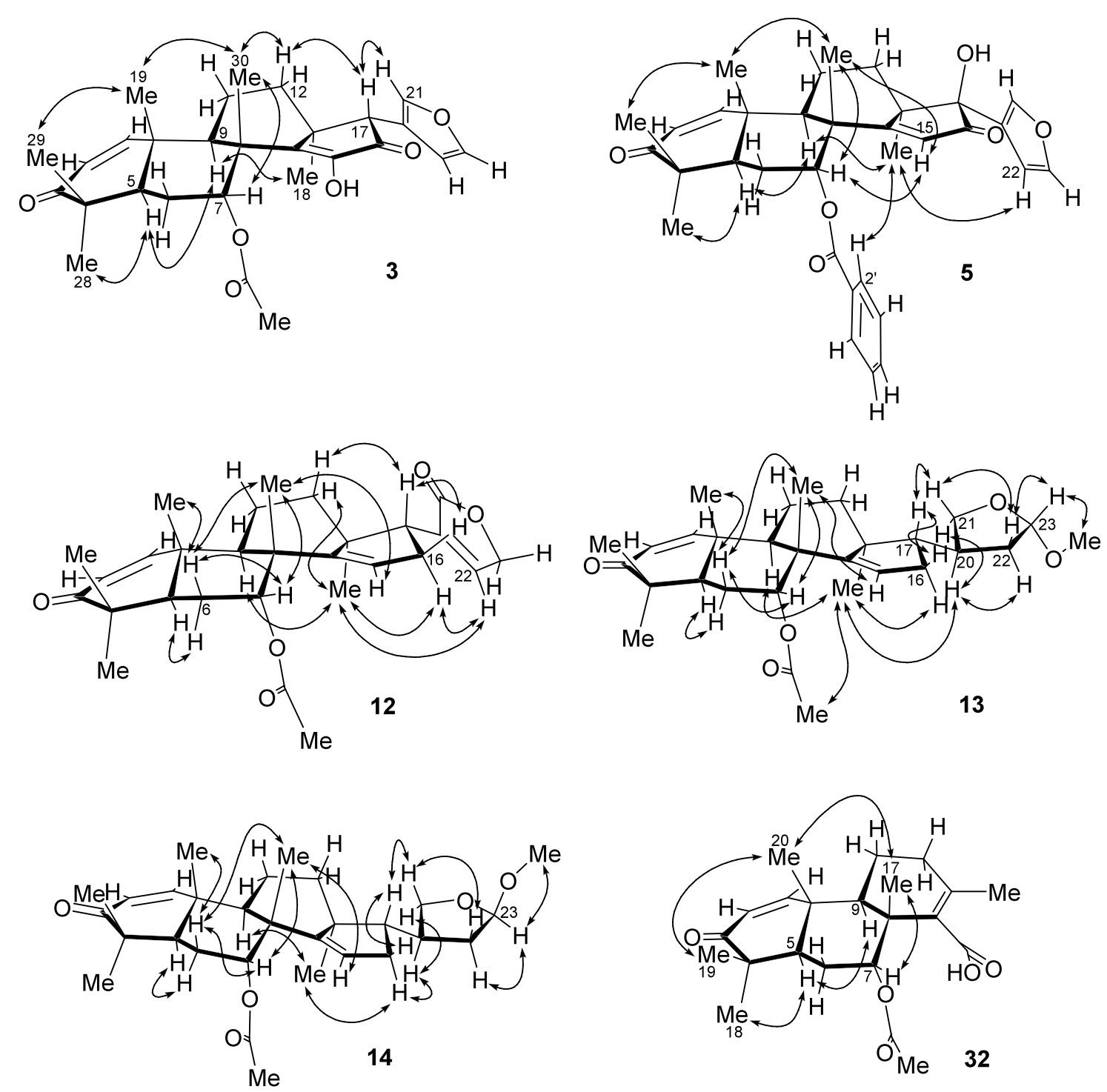

Fig. 2 Major NOE Correlations ( $\leftrightarrow$ ) for Compounds 3, 5, 12-14, and 32. ${ }^{28)}$

$\mathrm{V}_{\max } 1745 \mathrm{~cm}^{-1}$ for $\alpha, \beta$-unsaturated $\gamma$-lactone $\mathrm{e}^{10}$. This suggested the presence of the $\gamma$-lactone group attached to $\mathrm{C}$ 17 in 12 which was supported by the ${ }^{13} \mathrm{C}$ (Table 1$)\left[\delta_{\mathrm{C}} 134.2\right.$ (s, C-20), 174.2 (s, C-21), 146.4 (d, C-22), 70.2 (t, C-23)] and ${ }^{1} \mathrm{H}$ NMR spectra (Table 2) $\left[\delta_{\mathrm{H}} 7.22(1 \mathrm{H}, \mathrm{dd}, J=1.7,3.1 \mathrm{~Hz} ; \mathrm{H}-\right.$ 22 ) and 4.82 and 4.86 (each $1 \mathrm{H}$ and dt, $J=18.0,1.0 \mathrm{~Hz} ; \mathrm{H}-$ 23) $]^{10)}$. All the assignments of ${ }^{13} \mathrm{C}$ and ${ }^{1} \mathrm{H}$ NMR spectra of 12 were confirmed by DEPT, ${ }^{1} \mathrm{H}^{-1} \mathrm{H}$ COSY, HMQC, and HMBC spectra. Hence, the structure of 12 was $7 \alpha$-acetoxy-21,23epoxy-24,25,26,27-tetranorapotirucalla-1,14,20(22)-triene3,21-dione (23-deoxyazadironolide). The NOESY experiment (Fig. 2) and molecular modeling ${ }^{30}$ supported the relative configuration of 12 .

The molecular formula of 13 was determined to be
$\mathrm{C}_{29} \mathrm{H}_{42} \mathrm{O}_{5}$ from its HR-ESIMS ([M $\left.+\mathrm{H}\right]^{+}, m / z$ 471.3100). The IR spectrum showed absorption bands for ester carbonyl, conjugated cyclohexenone, and trisubstituted double bond groups. The ${ }^{13} \mathrm{C}$ (Table 1) and ${ }^{1} \mathrm{H}$ NMR spectra (Table 2) exhibited the presence of 5 tertirary methyls, one acetate methyl, one oxymethine, and the ring-A 1-en-3-one system. These suggested that compound 13 possesses the tetracyclic skeleton and substituents identical to those of compound 12 . The side-chain of 13 was indicated to formulate an acetal structure ${ }^{31)}$ with a methoxyl group $\left(\delta_{\mathrm{C}} 54.6, \delta_{\mathrm{H}}\right.$ 3.33) at $\mathrm{C}-23$ by analysis of ${ }^{1} \mathrm{H}-{ }^{1} \mathrm{H}$ COSY, HMQC, and HMBC spectra. Thus, diagnostic HMBC cross-correlations were observed for $\mathrm{H}-23$ with $\mathrm{C}-20, \mathrm{C}-21$, and $\mathrm{OCH}_{3}-$ 23 , and $\mathrm{OCH}_{3}-23$ with $\mathrm{C}-23$. Further, the stereochemistry 
of the acetal side-chain moiety was established by NOESY spectral analysis (Fig. 2) and molecular modeling ${ }^{30}$. The NOESY spectrum of 13 exhibited significant NOE correlations between [H-18 (13 $\alpha-\mathrm{Me})-\mathrm{H}-16 \alpha$; H-18-H-20-H$21 \alpha$ (pro-S); and $\mathrm{H}-20-\mathrm{H}-22 \alpha($ pro- $R)]$ on the $\alpha$-face of the molecule, and $[\mathrm{H}-16 \beta-\mathrm{H}-17-\mathrm{H}-21 \beta($ pro- $R)-\mathrm{H}-22 \beta($ pro- $S)-\mathrm{H}-$ $23]$ on the $\beta$-face of the molecule which suggested that the side-chain at C-17 is $\alpha$-oriented with $17 \mathrm{~S}^{-}$and $20 \mathrm{~S}$-chirarities and $S$-chirality at C-23 by orienting the methoxyl group down from the acetal ring plane on the global minimum energy conformation of $13^{30}$. These NOE correlations were confirmed by one-dimensional difference NOE experiment. Hence, the structure of 13 was elucidated as $(20 S, 23 S)$ - $7 \alpha$-acetoxy-21,23-epoxy-23-methoxy-24,25,26,27tetranorapotirucalla-1,14-dien-3-one, which we named limocin E.

Compound 14 gave a $[\mathrm{M}+\mathrm{H}]^{+}$ion in the HR-ESIMS at $m / z$ 471.3093, consistent with molecular formula $\mathrm{C}_{29} \mathrm{H}_{42} \mathrm{O}_{5}$. The UV and IR spectra, and ${ }^{13} \mathrm{C}$ (Table 1) and ${ }^{1} \mathrm{H}$ NMR spectra (Table 2) of 14 were very similar to those of 13, and the former was assigned to possess the same planar structure as that of the latter by the analysis of ${ }^{1} \mathrm{H}^{1} \mathrm{H}$ COSY, HMQC, and HMBC spectra. The NOESY spectrum of 14 showed significant NOE correlations (Fig. 2) between [H-18 $(13 \alpha-\mathrm{Me})-\mathrm{H}-16 \alpha-\mathrm{H}-20-\mathrm{H}-21 \alpha($ pro-S $)$ and H-22 $\alpha$ (pro- $R$ )-H$23]$ on the $\alpha$-face of the molecule, and [H-16 $\beta-\mathrm{H}-17-\mathrm{H}-$ $21 \beta($ pro- $R)-\mathrm{H}-22 \beta($ pro-S $)]$ on the $\beta$-face of the molecule suggesting that the side-chain at $\mathrm{C}-17$ is $\alpha$-oriented with $17 \mathrm{~S}$ and $20 S$-chirarities while $R$-chirarity at C-23 by orienting the methoxyl group up from the acetal ring plane on the global minimum energy conformation of $14^{30}$. These NOE correlations were confirmed by one-dimensional difference NOE experiment. Thus, compound 14 was the stereoisomer at C-23 of compound 13, and possesses the structure $(20 S, 23 R)$ - $7 \alpha$-acetoxy-21,23-epoxy-23-methoxy-24,25,26,27tetranorapotirucalla-1,14-dien-3-one (23-epilimocin E).

Compound 32 was assigned the molecular formula $\mathrm{C}_{22} \mathrm{H}_{30} \mathrm{O}_{5}$ from its HR-ESIMS ([M $\left.+\mathrm{Na}\right]^{+}, m / z$ 397.1989). Its IR spectrum exhibited absorption bands for an ester carbonyl, carboxyl ( $\left.\mathrm{v}_{\max } 1723 \mathrm{~cm}^{-1}\right)$, and conjugated cyclohexenone groups. The ${ }^{13} \mathrm{C}$ (Table 1 ) and ${ }^{1} \mathrm{H}$ NMR spectra (Table 2) exhibited the presence of 4 tertiary methyls, one acetate methyl, one vinyl methyl, one carboxyl, one oxymethine, one tetrasubstituted double bond, and the ring-A 1en-3-one system. The above evidence, coupled with a NMR comparison with compound $1^{6,28}$, and analysis of ${ }^{1} \mathrm{H}-{ }^{1} \mathrm{H}$ COSY, HMQC, HMBC, and NOESY (Fig. 2) spectra, confirmed that 32 was a diterpene acid possessing an isocopalane-type skeleton, i.e., $7 \alpha$-acetoxy-3-oxoisocopala-1,13dien-15-oic acid. This diterpene is corresponded to a ringD cleavage product of azadirone- and azadiradione-type limonoids.

All of the 32 compounds isolated from A. indica seeds were evaluated for their melanogenesis inhibitory activity.
By addition of these compounds at the concentration of 25 $\mu \mathrm{g} / \mathrm{mL}$ to an incubation medium of the B16 melanoma cell, all of the compounds, except for 16 and 21, inhibited the melanogenesis with the melanin content 2.9-66.9\% (Table 3). These were more inhibitive than a reference arbutin (melanin content $76.4 \%$ ) which has been recognized as a useful depigmentation compound for skin whitening in the cosmetic industry ${ }^{32}$. Although most of the inhibitory activity of these compounds is thought to be due to their cytotoxic action since they reduced significantly cell viability $(1.1-73.1 \%$ of cell viability at $25 \mu \mathrm{g} / \mathrm{mL}), 5$ compounds, including one gedunin-type (20) and 2 nimbin-type (26 and 27) limonoids, and 2 degraded limonoids (enneanortriterpenoids) (29 and 31), showed remarkable inhibition of melanogenesis (melanin content $9.5-26.0 \%$ at $25 \mu \mathrm{g} / \mathrm{mL}$ ) with no or almost no toxicity to the cells because cell viabilities were $85.8-108.1 \%$.

Seven compounds, $1,6,9,10,18,20$, and 21 , were evaluated with respect to their anti-inflammatory activity against TPA-induced inflammation in mice, and the inhibitory effects were compared with that of a commercially available anti-inflammatory drug, indomethacin. As shown in Table 4, all of the compounds, except for compound 26, exhibited marked anti-inflammatory activity with a 50\% inhibitory dose $\left(\mathrm{ID}_{50}\right)$ of $0.09-0.26 \mathrm{mg} /$ ear, which being more inhibitory than indomethacin $\left(\mathrm{ID}_{50}=0.30 \mathrm{mg} / \mathrm{ear}\right.$ ).

The inhibitory effect on EBV-EA activation induced by TPA was further examined as a preliminary evaluation of the potential anti-tumor-promoting effects ${ }^{33)}$ for all of the 32 compounds. The results are shown in Table 4, together with comparable data for retinoic acid (one of the retinoids that has been shown to have chemopreventive activity for a number of tumors ${ }^{34)}$.). All compounds tested allowed high viability (60-70\%) of Raji cells even at $32 \mathrm{nmol}$ (mol ratio of compound to TPA $=1000: 1$ ), indicating their low cytotoxicity to the cells at this high concentration. Each compound tested showed an inhibitory effect, with an $\mathrm{IC}_{50}$ value (concentration of $50 \%$ inhibition with respect to positive control) of 230-501 mol ratio/32 pmol TPA. As such, these compounds were more potent than or almost equipotent to the reference retinoic acid (482 mol ratio/32 pmol TPA). Of the compounds tested, compounds $6,10,21-25,29$, and 31 (318-398 mol ratio/32 pmol TPA), and especially an isocopalane-type diterpenoid, 32 (230 mol ratio/32 pmol TPA), exhibited more potent inhibitory effects than the others. Since the inhibitory effects on EBV-EA induction have been demonstrated to correlate with those against tumor promotion in vivo ${ }^{33)}$, these compounds may be considered potential anti-tumor-promoters.

Subsequently, we evaluated the inhibitory effect of compound 21 in a tumor model in mouse skin. The incidence (\%) of papilloma-bearing mice and the average numbers of papillomas per mouse in a two-stage carcinogenesis test in mouse skin using peroxynitrite (ONOO-; PN) as an initiator 
Table 3 Melanogenesis Inhibitory Activities and Cytotoxicities in B16 Mouse Melanoma Cells of the Extracts of Azadirachta indica Seeds and the Compounds Isolated from the Extracts.

\begin{tabular}{|c|c|c|c|}
\hline & \multirow[b]{3}{*}{ compound } & \multirow{2}{*}{\multicolumn{2}{|c|}{$\begin{array}{c}\text { melanogenesis inhibitory activity and } \\
\text { cytotoxicity }\end{array}$}} \\
\hline & & & \\
\hline & & melanin content $\mathrm{t}^{\mathrm{b})}$ & cell viabilityb) \\
\hline \multicolumn{2}{|c|}{ control (100\% DMSO) } & $100.0 \pm 1.35$ & $100.0 \pm 2.50$ \\
\hline 1 & azadiradione & $5.1 \pm 0.79$ & $15.5 \pm 0.83$ \\
\hline 2 & 7-benzoylnimbocinol & $4.8 \pm 0.68$ & $8.8 \pm 0.58$ \\
\hline 3 & 15-hydroxyazadiradione & $15.0 \pm 0.62$ & $30.1 \pm 0.46$ \\
\hline 4 & 17-hydroxyazadiradione & $19.1 \pm 1.33$ & $65.8 \pm 4.43$ \\
\hline 5 & 7-benzoyl-17-hydroxynimbocinol & $8.3 \pm 1.58$ & $3.5 \pm 0.15$ \\
\hline 6 & epoxyazadiradione & $2.9 \pm 0.52$ & $2.2 \pm 0.07$ \\
\hline 7 & 7-deacetyl-7-benzoylepoxyazadiradione & $4.6 \pm 0.03$ & $1.1 \pm 0.04$ \\
\hline 8 & 17-epiazadiradione & $12.8 \pm 0.42$ & $4.0 \pm 0.13$ \\
\hline 9 & 17-epi-17-hydroxyazadiradione & $9.0 \pm 1.44$ & $30.2 \pm 0.96$ \\
\hline 10 & 7-acetyl-16,17-dehydro-16-hydroxyneotrichilenone & $6.8 \pm 0.49$ & $59.6 \pm 1.28$ \\
\hline 11 & azadiradionolide & $31.6 \pm 1.21$ & $68.5 \pm 1.57$ \\
\hline 12 & 23-deoxyazadironolide & $6.4 \pm 0.29$ & $15.7 \pm 0.76$ \\
\hline 13 & $\operatorname{limocin} \mathrm{E}$ & $10.9 \pm 1.25$ & $3.7 \pm 0.12$ \\
\hline 14 & 23-epilimocin E & $9.4 \pm 1.76$ & $7.1 \pm 0.13$ \\
\hline 15 & 20,21,22,23-tetrahydro-23-oxoazadirone & $10.4 \pm 0.42$ & $30.2 \pm 1.21$ \\
\hline 16 & 3-acetyl-7-tigloylvilasinin lactone & $84.8 \pm 0.78$ & $68.0 \pm 1.08$ \\
\hline 17 & gedunin & $7.4 \pm 0.39$ & $12.6 \pm 0.43$ \\
\hline 18 & 7-deacetylgedunin & $6.5 \pm 0.03$ & $21.6 \pm 0.86$ \\
\hline 19 & 7-deacetyl-7-benzoylgedunin & $5.2 \pm 0.54$ & $5.9 \pm 0.44$ \\
\hline 20 & nimolicinol & $9.5 \pm 0.20$ & $97.8 \pm 3.09$ \\
\hline 21 & azadirachtin B & $101.6 \pm 1.42$ & $89.2 \pm 1.24$ \\
\hline 22 & 11-epiazadirachtin D & $36.2 \pm 1.81$ & $68.7 \pm 2.27$ \\
\hline 23 & vepaol & $43.7 \pm 1.88$ & $71.4 \pm 2.27$ \\
\hline 24 & 23-epivepaol & $33.5 \pm 0.08$ & $67.5 \pm 1.83$ \\
\hline 25 & 3-acetyl-11-methoxy-1-tigloylazadirachtinin & $66.9 \pm 1.54$ & $55.8 \pm 0.99$ \\
\hline 26 & nimbin & $20.9 \pm 1.05$ & $96.3 \pm 2.87$ \\
\hline 27 & 6-deacetylnimbin & $26.0 \pm 1.40$ & $91.9 \pm 2.74$ \\
\hline 28 & 6-acetylnimbandiol & $49.9 \pm 7.39$ & $73.1 \pm 1.25$ \\
\hline 29 & $\alpha$-nimolactone & $18.3 \pm 1.00$ & $108.1 \pm 4.34$ \\
\hline 30 & $\beta$-nimolactone & $6.1 \pm 0.67$ & $53.9 \pm 1.50$ \\
\hline 31 & desfuranoazadiradione & $13.6 \pm 1.08$ & $85.8 \pm 3.25$ \\
\hline 32 & $7 \alpha$-acetoxy-3-oxoisocopala-1,13-dien-15-oic acidarbutin & $10.8 \pm 1.02$ & $75.5 \pm 1.50$ \\
\hline \multicolumn{2}{|c|}{$\operatorname{arbutin}^{\mathrm{c})}$} & $85.2 \pm 5.35$ & $100.1 \pm 4.80$ \\
\hline
\end{tabular}

a) Sample concentration determined was $25 \mu \mathrm{g} / \mathrm{mL}$. Melanin contents (\%) and cell viability (\%) were determined based on the absorbances at 420 and 570-620 nm, respectively, by comparison with those for DMSO (100\%). The absorbances at 420 and 570-620 nm of DMSO were 0.523 and 0.520, respectively.

b) Values of fourfold experiments. Concentration of DMSO in the sample solution was $5 \mu \mathrm{L} / \mathrm{mL}$.

c) Reference compound. 
Table 4 Inhibitory Effects of Compounds from Azadirachta indica Seeds and Reference Compounds on TPA-induced Inflammation in Mice and on the Induction of Epstein-Barr Virus Early Antigen.

\begin{tabular}{|c|c|c|c|c|c|c|}
\hline \multirow[b]{3}{*}{ compound } & \multirow{3}{*}{$\begin{array}{c}\begin{array}{c}\text { inhibition of } \\
\text { inflammation }\end{array} \\
\begin{array}{c}\mathrm{ID}_{50}^{\mathrm{a})} \\
(\mathrm{mg} / \text { ear })\end{array}\end{array}$} & \multicolumn{4}{|c|}{ percentage of EBV-EA induction ${ }^{\text {b) }}$} & \multirow{3}{*}{$\begin{array}{c}\left.\mathrm{IC}_{50} \mathrm{c}\right) \\
(\mathrm{mol} \mathrm{ratio} / 32 \\
\text { pmol TPA })\end{array}$} \\
\hline & & \multicolumn{4}{|c|}{ concentration (mol ratio/ 32 pmol TPA) } & \\
\hline & & 1000 & 500 & 100 & 10 & \\
\hline 1 & 0.09 & $13.8(70)$ & 63.2 & 81.2 & 100 & 498 \\
\hline 2 & & $17.3(70)$ & 65.3 & 83.2 & 100 & 501 \\
\hline 3 & & $10.3(60)$ & 47.3 & 75.9 & 100 & 465 \\
\hline 4 & & $12.3(60)$ & 49.7 & 75.3 & 100 & 477 \\
\hline 5 & & $11.8(60)$ & 52.1 & 81.6 & 100 & 475 \\
\hline 6 & 0.17 & $9.5(70)$ & 33.6 & 80.6 & 100 & 372 \\
\hline 7 & & $13.1(60)$ & 53.8 & 82.9 & 100 & 482 \\
\hline 8 & & $13.7(60)$ & 49.9 & 76.8 & 100 & 479 \\
\hline 9 & 0.16 & $6.5(70)$ & 45.2 & 77.1 & 100 & 481 \\
\hline 10 & 0.26 & $7.1(70)$ & 30.4 & 79.2 & 100 & 369 \\
\hline 11 & & $11.2(60)$ & 49.9 & 74.1 & 100 & 471 \\
\hline 12 & & $9.7(70)$ & 50.1 & 80.6 & 100 & 487 \\
\hline 13 & & $11.9(60)$ & 51.3 & 81.0 & 100 & 473 \\
\hline 14 & & $11.0(60)$ & 51.1 & 80.2 & 100 & 470 \\
\hline 15 & & $9.0(70)$ & 48.9 & 79.1 & 100 & 484 \\
\hline 16 & & $0 \quad(60)$ & 44.3 & 75.0 & 98.3 & 393 \\
\hline 17 & & $10.0(70)$ & 58.6 & 78.4 & 100 & 492 \\
\hline 18 & 0.16 & $8.7(70)$ & 57.3 & 76.3 & 100 & 488 \\
\hline 19 & & $10.1(70)$ & 58.1 & 77.9 & 100 & 491 \\
\hline 20 & 0.12 & $12.3(70)$ & 60.6 & 80.6 & 100 & 493 \\
\hline 21 & & $0 \quad(70)$ & 41.5 & 74.1 & 98.3 & 384 \\
\hline 22 & & $0 \quad(70)$ & 42.9 & 73.6 & 97.9 & 382 \\
\hline 23 & & $0 \quad(70)$ & 41.0 & 71.0 & 96.5 & 379 \\
\hline 24 & & $0 \quad(70)$ & 41.3 & 72.4 & 97.4 & 380 \\
\hline 25 & & $0 \quad(70)$ & 44.7 & 76.2 & 99.2 & 398 \\
\hline 26 & 0.51 & $14.2(60)$ & 63.4 & 81.6 & 100 & 499 \\
\hline 27 & & $4.7(70)$ & 46.2 & 88.8 & 100 & 489 \\
\hline 28 & & $3.2(70)$ & 44.3 & 76.2 & 100 & 406 \\
\hline 29 & & $0 \quad(70)$ & 24.7 & 71.1 & 95.4 & 318 \\
\hline 30 & & $11.5(60)$ & 50.0 & 79.8 & 100 & 471 \\
\hline 31 & & $3.8(70)$ & 28.6 & 75.9 & 100 & 359 \\
\hline 32 & & $1.3(60)$ & 23.6 & 71.0 & 95.3 & 230 \\
\hline indomethacind) & 0.30 & & & & & \\
\hline retinoic acidd) & & $21.6(60)$ & 49.3 & 76.3 & 100 & 482 \\
\hline
\end{tabular}

a) $\mathrm{ID}_{50}: 50 \%$ inhibitory dose.

b) Values represent percentages relative to the positive control value. TPA ( $32 \mathrm{pmol}, 20 \mathrm{ng})=100 \%$. Values in parentheses are viability percentages of Raji cells.

c) $\mathrm{IC}_{50}$ represents the molar ratio to TPA that inhibits $50 \%$ of positive control (100\%) activated with 32 pmol of TPA.

d) Reference compound. 
and TPA as a promoter are presented in Figs. $3 \mathrm{~A}$ and $3 \mathrm{~B}$, respectively. In the positive control group, the first papilloma appeared after 7 weeks, and the incidence of the papilloma bearing mice was 100\% after 14 weeks (Fig. 3A). Oral administration of 21 exhibited a significant inhibitory effect in the PN-induced and TPA-promoted experiment in mice. When $21(780 \mathrm{nmol})$ in drinking water was administered, from one week before to one week after the initiation treatment, whereas the first papilloma appeared after 9 weeks, the incidence of the papilloma bearing mice was $87 \%$ even after 20 weeks of promotion. In the average number of papillomas per mouse (Fig. 3B), 21 reduced the number of papillomas compared to the control group. Whereas $c a .7$ papillomas per mouse were observed after 20 weeks of promotion in group I, only ca. 4 papillomas were observed after the same period of promotion in group II. From these results, 21 appears effective for the inhibition of $\mathrm{PN}$-initiated carcinogenesis on mouse skin. PN (ONOO-), which is produced by the reaction of $\mathrm{NO}$ with superoxide, is a potent tumor-initiating agent ${ }^{35}$, as well as an oxidant, and nitrating and hydroxylating agent. Compound 21 might be suggested to intercept, and neutralize, potent chemical carcinogens, such as reactive oxygen species (ROS; superoxide, and peroxy and hydroxyl radicals) and NO donors.

Our data suggest that some of the nortriterpenoid (limonoid) and diterpenoid constituents of $A$. indica seeds are useful as skin whitening agents, anti-inflammatory agents, and cancer chemopreventive agents.

\section{ACKNOWLEDGEMENT}

This work was supported, in part, by a grant "Academic Frontier" Project for Private Universities and a matching fund subsidy from MEXT (Ministry of Education, Culture, Sports, Science and Technology), 2007-2010, and by a Grant-in-Aid from the Japan Society for the Promotion of Science (No. 20590015), 2008-2010.

\section{References and Notes}

1. Van der Nat, J.M.; Van der Sluis, W.G.; De Silva, K.T.; Labadie, R.P. Ethnopharmacognostical survey of Azadirachita indica A. Juss (Meliacea). J. Ethnopharmacol. 35, 1-24 (1991).

2. Biswas, K.; Chattopadhyay, I.; Banerjee, R.K.; Bandyopadhyay, U. Biological activities and medicinal properties of neem (Azadirachta indica). Curr. Sci. 82, 1336-1345 (2002).

3. Akhila, A.; Rani, K. Chemistry of the neem tree (Azadirachta indica A. Juss.). Fortschr. Chem. Org. Naturst. 78, 47-149 (1999).

4. Brahmachari, G. Neem - An omnipotent plant: A retrospection. Chem. Biochem. 5, 408-421 (2004).

5. Akihisa, T.; Yasukawa, K. Anti-Inflammatory and antiallergic properties of triterpenoids from plants. in Biomaterials from Aquatic and Terrestrial Organisms. (Fingerman, M.; Nagabhushanam R. ed.) Science Publ. Enfield, pp. 63-114 (2006).

6. Siddiqui, B.S.; Faizi, S.; Siddiqui, S. Triterpnoids from the fresh fruit coats of Azadirachta indica. Phytochem 31, 4275-4278 (1992).

7. Kraus, W.; Cramer, R.; Sawitzki, G. Tetranortriter-
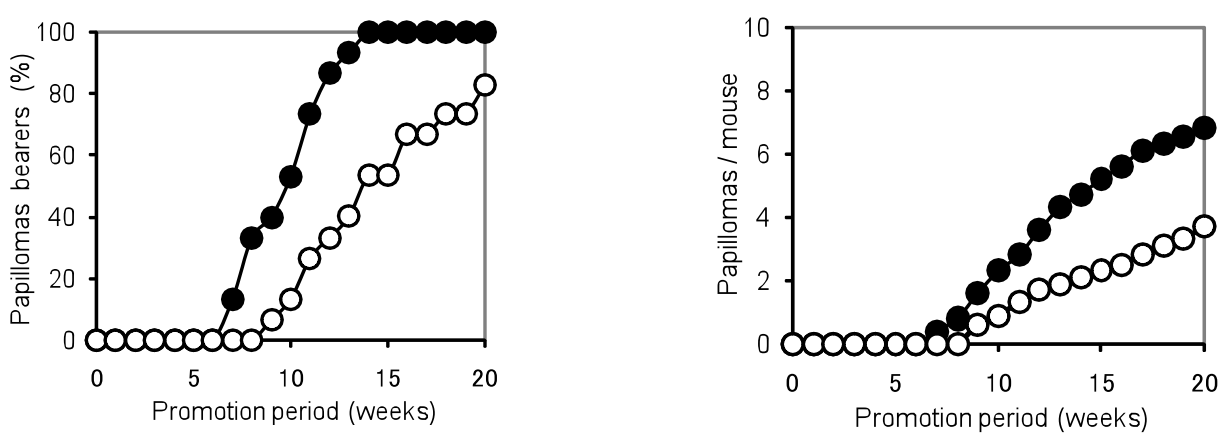

Fig. 3 Inhibitory Effect of Azadirachtin B (21) on Mouse Skin Carcinogenesis Induced by PN and TPA.

A: percentage of mice bearing papillomas; B: average number of papillomas per mouse. Positive control, PN (390 nmol) + TPA (1.7 nmol) alone (group I; $n=$ 15); $\bigcirc:$ PN (390 nmol) + azadirachtin B (21) (780 nmol; 2 weeks) + TPA (1.7 nmol) (group II; $n=15$ ). At 20 weeks of promotion, the number of papillomas per mouse differed significantly $(P<0.01$, using Student's $t$-test $)$ between groups I and II. The number of papillomas per mouse for each group was $6.8(\mathrm{SD}= \pm 1.6)$ and $3.7(\mathrm{SD}= \pm 1.4)$ for groups I and II, respectively. 
penoids from the seeds of Azadirachta indica. Phytochemi. 20, 117-120 (1981).

8. Kraus, W.; Cramer, R. 17-Epi-azadiradion und 17- $\beta$ Hydroxy-azadiradion, Zwei Neue Inhaltsstoffe aus Azadirachta indica A. Juss. Tetrahedron Lett. 19, 2395-2398 (1978).

9. Lavie, D.; Levy, E.C. Meliane_meliacin relationship. Tetrahedron 27, 3941-3947 (1971).

10. Siddiqui, B.S.; Ghiasuddin; Faizi, S.; Rasheed, M. Triterpenoids of the fruit coats of Azadirachta indica. J. Nat. Prod. 62, 1006-1009 (1999).

11. Paula, J.R.; Vieira, I.J.C.; Silva, M.F.G.F.; Fo, E.R.; Fernandes, J.B.; Vieira, P.C.; Pinheiro, A.L.; Vilela, E.F. Sesquiterpenes, triterpenoids, limonoids and flavonoids of Cedrela odorata graft and speculations on the induced resistance against Hypsipyla grandella. Phytochem. 44, 1449-1454 (1997).

12. Bilton, J.N.; Broughton, H.B.; Jones, P.S.; Ley, S.V.; Lidert, Z.; Morgan, E.D.; Rzepa, H.S.; Sheppart, R.N.; Slawin, A. M.Z.; Williams, D.J. An X-ray crystallographic, mass spectroscopic, and NMR study of the limonoid insect antifeedant azadirachtin and related derivatives. Tetrahedron 43, 2805-2815 (1987).

13. Khalid, S.A.; Duddeck, H.; Sierra, M.G. Isolation and characterization of an antimalarial agent of the neem tree Azadirachta indica. J. Nat. Prod. 52, 922_927 (1989).

14. Mitsui, K.; Saito, H.; Yamamura, R.; Fukaya, H.; Hitotsuyanagi, Y.; Takeya, K. Hydroxylated gedunin derivatives from Cedrela sinensis. J. Nat. Prod. 69, 1310-1314 (2006).

15. Siddiqui, S.; Faizi, S.; Siddiqui, B.S. Studies on the chemical constituents of Azadirachta indica A. Juss (Meliaceae) Part I: Isolation and structure of a new tetranortriterpenoid - nimolicinol. Heterocycles 22, 295-298 (1984).

16. Klenk, A.; Bokel, M.; Kraus, W. 3-Tigloylazadirachtol (tigloyl $=2$-methylcrotonoyl), an insect growth regulating constituent of Azadirachta indica. J. Chem. Soc., Chem. Commun. 523-524 (1986).

17. Ramji, N.; Venkatakrishnan, K.; Madyastha, K.M. 11Epi-azadirachtin D: an epimeric azadirachtin analogue from Azadirachta indica. Phytochem. 49, 265-267 (1998).

18. Denholm, A.A.; Jennens, L.; Ley, S.V.; Wood, A. Chemistry of insect antifeedants from Azadirachta Indica (Part 19): A potential relay route for the synthesis of azadirachtin. Tetrahedron 51, 6591-6604 (1995).

19. Kraus, W.; Bokel, M.; Bruhn, A.; Cramer, R.; KIlaiber, I.; Klenk, A.; Nagl, G.; Pöhnl, H.; Sadlo, H.; Vogler, B. Structure determination by $\mathrm{nmr}$ of azadirachtin and related compounds from Azadirachta indica A. Juss (Meliaceae). Tetrahedron 43, 2817-2830 (1987).

20. Jarvis. A.P.; Johnson, S.; Morgan, E.D.; Simmonds,
M.S.J.; Blaney, W.M. Photooxidation of nimbin and salannin, tetranortriterpenoids from the neem tree (Azadirachta indica). J. Chem. Ecol. 23, 2841-2860 (1997).

21. Johnson, S.; Morgan, E.D. Comparison of chromatographic systems for triterpenoids from neem (Azadirachta indica) seeds. J. Chromatogr. A 761, 53-63 (1997).

22. Kraus, W.; Cramer, R. Pentanortriterpenoids from Azadirachta indica A. Juss (Meliaceae). Int. Conf. Chem. Biotechnol. Biol. Act. Nat. Prod. 3, 27-29 (1981).

23. Kraus, W.; Köll-Weber, M.; Maile, R.; Wunder, T.; Vogler, B. Biologically active constituents of tropical and subtropical plants. Pure Appl. Chem. 66, 2347-2352 (1994).

24. Butler, B.J.; Ellenberger, W.P.; Omilinsky, B.A. Interntl. Pat. WO 94/02019 (1994).

25. Akihisa, T.; Tokuda, H.; Yasukawa, K.; Ukiya, M.; Kiyota, A.; Sakamoto, N.; Suzuki, T.; Tanabe, N.; Nishino, H. Azaphilones, furanoisophthalides, and amino acids from the extracts of Monascus pilosus-fermented rice (red-mold rice) and their chemopreventive effects. $J$. Agric. Food Chem. 53, 562-565 (2005).

26. Akihisa, T.; Tokuda, H.; Ukiya, M.; Kiyota, A.; Yasukawa, K.; Sakamoto, Y.; Kimura, Y.; Suzuki, T.; Takayasu, J.; Nishino, H. Anti-tumor-initiating effects of monascin, an azaphilonoid pigment from the extract of Monascus pilosus fermented rice (red-mold rice). Chem. Biodivers 2, 1305-1309 (2005).

27. Akihisa, T.; Higo, N.; Tokuda, H.; Ukiya, M.; Akazawa, H.; Tochigi, Y.; Kimura, Y.; Suzuki, T.; Nishino, H. Cucurbitane-type triterpenoids from the fruits of Momordica charantia and their cancer chemopreventive effects. J. Nat. Prod. 70, 1233-1239 (2007).

28. Lee, S.M.; Olsen, J.I.; Schweizer, M.P.; Klocke, J.A. 7Deacetyl-17 $\beta$-hydroxyazadiradione, a new limonoid insect growth inhibitor from Azadirachta indica. Phytochem. 27, 2773-2775 (1988).

29. Breitmaier, E.; Voelter, W. Carbon-13 NMR Spectroscopy. 3rd, Completely revised ed. VCH Verlagsgesellschaft. Weinheim. Germany. p. 448 (1987).

30. Conformational analysis: Global minimum conformation search of compounds $3,5,12-14$, and 32 was done using CAChe CONFLEX with MM2 force field (CAChe version 5.5; Fujitsu Co., Tokyo, Japan).

31. Siddiqui, S.; Siddiqui, B.S.; Ghiasudddin; Faizi, S. Terpenoids from fruit coatings of Azadirachta indica. Phytochem. 30, 1615-1619 (1991).

32. Lim, Y.-J.; Lee, E.H.; Kang, T.H.; Ha, S.K.; Oh, M.S.; Kim, S.M.; Yoon, T.-J.; Kang, C.; Park, J.-H.; Kim, S.Y. Inhibitory effects of arbutin on melanin biosynthesis of $\alpha$-melanocyte stimulating hormone-induced hyperpigmentation in cultured brownish guinea pig skin tissues. Arch. Pharm. Res. 32, 367-373 (2009). 
33. Akihisa, T.; Yasukawa, K.; Tokuda, H. Potentially cancer chemopreventive and anti-inflammatory terpenoids from natural sources. in Studies in Natural Products Chemistry, Vol. 29, Bioactive Natural Products (Part J) (Atta-ur-Rahman, ed.). Elsevier Science B.V. Amsterdam. pp 73-126 (2003).

34. Niles, R.M. Biomarker and animal models for assess- ment of retinoid efficacy in cancer chemoprevention. Acta Pharmacol. Sin. 28, 1383-1391 (2007).

35. Sakurai, N.; Kozuka, M.; Tokuda, H.; Mukainaka, T.; Enjo, F.; Nishino, H.; Nagai, N.; Sakurai, Y.; Lee, K.-H. Cancer preventive agents. Part 1: Chemopreventive potential of cimigenol, cimigenol-3,15-dione, and related compounds. Bioorg. Med. Chem. 13, 1403-1408 (2005). 